\title{
Le cancer est-il une maladie nouvelle?
}

\author{
A propos du diagnostic des maladies tumorales \\ dans le De Medicina de Celse ${ }^{1}$
}

François Luthi

\section{Summary}

Cancer is a particularly common disease in modern societies. Moreover, epidemiology considers it typical of contemporary pathology. Nevertheless, the abundant ancient literature - in the De Medicina by Celsus, among others leads us to believe that numerous benign and malignant tumours were observed if not identified as such. Hence, it is possible that both the change in medical conceptualization and the real increase in the prevalence are responsible for the actual importance of cancer.

\section{Résumé}

Les cancers sont des maladies particulièrement fréquentes dans toutes les sociétés modernes. L'épidémiologie les considère d'ailleurs comme caractéristiques du paysage pathologique contemporain. Pourtant, l'abondance des descriptions littéraires antiques, notamment dans le De Medicina de Celse, laisse supposer que de nombreuses tumeurs bénignes et malignes ont été observées, sinon reconnues en tant que telles. Ainsi, il est possible d'imaginer que la transformation de la conceptualisation médicale, et non seulement l'augmentation réelle de la prévalence, est également responsable de l'importance actuelle du cancer.

1 D'après une communication présentée aux «Journées de la Société suisse d'Histoire de la médecine et des Sciences naturelles». Genève, le 12 octobre 1995.

F. Luthi, Clinique d'Orthopédie et de Chirurgie de l'Appareil Locomoteur, Département de Chirurgie, Hôpitaux Universitaires de Genève, rue Micheli-du-Crest 24, CH-1205 Genève 
Il y presque vingt siècles, Celse déclarait dans le De Medicina:

(V,28.2 A ) Ce n'est pas le même danger² qu'apporte le carcinome à moins qu'il n'ait été malmené par un médecin incompétent.

Aujourd'hui, un Européen ou un Américain sur trois ${ }^{3}$ présentera au cours de son existence au moins un cancer. Contrairement à certaines idées, la place de la maladie tumorale n'est guère moins inquiétante dans les pays du TiersMonde, puisque elle est responsable d'environ $13 \%$ des décès ${ }^{4}$. Ainsi, cette pathologie est-elle considérée à juste titre comme caractéristique du paysage pathologique contemporain. Que s'est-il donc produit au cours de ces deux millénaires?

Plusieurs hypothèses peuvent être avancées: d'abord, le carcinome de Celse pourrait ne pas correspondre au cancer moderne. La nomenclature médicale antique propose en effet déjà les termes de tumeur, cancer, carcinome, que nous utilisons toujours pour désigner les néoplasies. Si les deux premiers ne représentent que rarement des formations tumorales, le carcinome ainsi que les expressions qui en dérivent, par exemple «qui ressemble au carcinome», «qui a le comportement du carcinome» se rapportent le plus souvent à ce que nous appelons des tumeurs, même si l'idée de malignité et à fortiori celle de tumeur épithéliale maligne est complètement absente.

Une deuxième hypothèse serait d'imaginer que le comportement du cancer s'est modifié avec le temps, qu'il est devenu plus «méchant», ou au contraire que nous sommes devenus moins résistants, plus réceptifs que nos ancêtres? Evolution de la maladie ou réminiscence de l'Age d'Or, variation de la sensibilité humaine aux maladies, ces idées sont en contradiction avec les acquis de la paléopathologie qui montrent une permanence de la plupart des phénomènes biologiques, ceci au moins à vue humaine ${ }^{5}$.

La dernière hypothèse, celle qui est généralement reprise dans la littérature, souligne non seulement les transformations apparues depuis les temps anciens dans l'environnement humain et les connaissances médicales, mais aussi la modification du comportement de l'homme (p. ex. le tabagisme), toutes choses qui ont certainement augmenté l'impact de la maladie tumorale. Ainsi, l'opinion souvent admise attribue un caractère exceptionnel à la ma-

2 Le carcinome est comparé au carbunculus ( $\mathrm{V}, 28.1)$, maladie qui correspond principalement au charbon et qui est considérée par Celse comme une affection particulièrement dangereuse.

3 Andreoli TE, Bennett JC, Carpenter CCJ, Plum F, Smith HL, eds : Cecil Essentials of Medicine, Philadelphia, Saunders WB, $3^{e}$ éd, 1993.

4 Boffetta P, Parkin DM: Cancer in Developing Countries. CA Cancer J Clin 1994; 44: 81-90.

5 Grmek MD: Les maladies à l'aube de la civilisation occidentale, Paris, Payot, 1983. 
ladie tumorale avant l'époque moderne ${ }^{6}$. Si cela s'avérait exact, le cancer apparaîtrait effectivement comme une maladie nouvelle.

Mais qu'est-ce qu'une maladie nouvelle? Grmek a précisé plusieurs situations historiques qui intéressent notre discussion ${ }^{7}:$ «1) on découvre une maladie parce que l'art de l'observation et de la conceptualisation médicale s'est amélioré; 2) on constate la présence, dans un pays ou plutôt dans une aire culturelle, d'une maladie qui y était jusqu'alors inconnue mais qu'on avait déjà observée ailleurs; 3) on observe une maladie qu'on n'avait jamais vue, dont on avait jamais entendu parler et qui pourtant, si elle avait existé, serait difficilement passée inaperçue.»

Quels sont les moyens à notre disposition pour déterminer si le cancer appartient ou non à l'un de ces exemples?

L'étude des anciens traités de médecine est une de nos sources principales. Parmi ceux-ci, le De Medicina de Celse présente un panorama complet du savoir médical au début du I ${ }^{\text {er }}$ s. apr. J.-C. Afin de rechercher l'ensemble des tableaux cliniques compatibles avec une maladie tumorale, les huit livres qui composent le De Medicina ont été analysés selon les hypothèses suivantes:

1. La tumeur a été reconnue ou suspectée.

2. La tumeur n'a pas été reconnue ou suspectée, mais la description clinique l'évoque.

3. La tumeur n'est pas décrite, mais un symptôme pathognomonique ou un signe d'appel est proposé.

Tous les passages choisis ont ensuite été proposés sans commentaire ni diagnostic à l'opinion de deux médecins ${ }^{8}$, qui ont réalisé séparément un diagnostic différentiel. Les textes pour lesquels au moins un diagnostic tumoral a obtenu l'unanimité ont été retenus. Le commentaire de l'ensemble de ces passages, qui constitue un travail de thèse ${ }^{9}$, a permis de réaliser un fichier des tumeurs. L'ensemble des systèmes d'organes y est représenté. Un diagnostic différentiel aussi étendu que possible est systématiquement proposé, car l'exercice du diagnostic rétrospectif est périlleux et il est toujours difficile de

6 Grmek MD: Paléopathologie des tumeurs osseuses malignes. Histoire des sciences médicales 1975-1976; 9: 21-50.

7 Grmek MD: La dénomination latine des maladies considérées comme nouvelles par les auteurs antiques. In: Sabbah G, éd.: Mémoire X du centre Jean Palerme: Le latin médical, la constitution d'un langage scientifique. St-Etienne, Publications de l'université de St-Etienne, 1991: 195-214.

8 Dr Pierre Kaeser, médecin-adjoint (Hôpital des Cadolles, Neuchâtel), Dr Robert Munger, médecin-chef (Hôpital du Locle), médecin-adjoint (Hôpital de La Chaux-de-Fonds).

9 Luthi F: Les tumeurs dans l'œuvre de Celse: diagnostic, diagnostic différentiel et comparaison avec les témoignages archéologiques; Med Diss Lausanne, 1996. 
prouver la description d'une maladie particulière. Plus de 70 passages ont été étudiés. Il est naturellement impossible de donner ici un reflet exhaustif de l'ensemble des pathologies retenues.

Dans un certain nombre de situations, il est plausible de considérer que Celse a suspecté l'origine de la maladie. Ces textes contiennent généralement les termes «carcinoma», «quae fere carcinodes est», «quasi carcinoma», «caro quoque carcinomati similis». Il s'agit généralement de tumeurs externe ${ }^{10} \mathrm{ou}$ qui se sont extériorisées (peau, sein, organes génitaux, sphère ORL). Il n'est en revanche pas imaginable de se fier dans une telle recherche aux mots latins «tumor» ou «cancer», qui ne sont pas synonymes de leurs équivalents modernes. Dans la majorité des passages retenus, c'est le tableau clinique qui évoque la néoplasie, mais seulement à la lumière des connaissances actuelles. A une seule reprise, c'est un symptôme pathognomonique qui affirme le diagnostic (adénome hypophysaire ${ }^{11}$ ).

Afin d'illustrer ce propos, trois textes ont été choisis qui sont brièvement exposés. De ces exemples, le premier a été reconnu comme tumoral, alors que les autres appartiennent à la deuxième situation - lorsque la tumeur n'a pas été reconnue - celle qui est la plus fréquente; mais aussi celle qui est la plus utile pour cerner le concept cancer - maladie nouvelle. A chaque fois, une néoplasie peut être considérée comme une hypothèse diagnostique sérieuse, mais avec une certitude plus ou moins grande selon les cas.

Le premier exemple concerne l'ORL, le second l'ophtalmologie et le dernier la gastroentérologie ${ }^{12}$.

\section{Premier texte:}

(VI, 8.2 A B) Parfois dans les narines naissent des espèces de morceaux de chair semblables aux pointes des seins des femmes et ils adhèrent par leurs parties inférieures qui sont très charnues. Il faut les soigner par des médicaments caustiques sous l'effet desquels ils sont complètement détruits. Mais le polype est un bout de chair tantôt blanc, tantôt rougeâtre qui adhère à l'os du nez; tantôt allant vers les lèvres, il remplit les narines, et tantôt allant en arrière à travers cette ouverture par laquelle le souffle descend des narines jusqu'à la gorge, il grandit au point qu'on peut le voir derrière la luette et il étrangle le malade, surtout quand souffle l'auster (vent du sud) ou l'eurus (vent de l'est); d'ordinaire ce bout de chair est mou rarement dur, et quand il est dur, il gêne davantage le souffle et dilate le nez. D'ordinaire, il est semblable au carcinome, raison pour laquelle il ne doit pas être touché.

$10 \mathrm{La}$ connaissance de tumeurs internes par Celse est une controverse qui repose sur les différentes leçons possibles du texte (V,28.2 A).

11 Livre II, 8. 41. Il s'agit de l'adénome à prolactine.

12 Les traductions ont été réalisées en collaboration avec M. le Professeur Ph. Mudry, Université de Lausanne, Faculté des Lettres, section des sciences de l'Antiquité, Latin. 
Il s'agit donc de la description de formations charnues à l'intérieur des fosses nasales. Il en existe deux catégories: les caroncules - «petits morceaux de chair» - et les polypes. Les premières représentent sans doute les polypes au sens moderne du terme, pseudo-tumeurs inflammatoires. Les seconds ne peuvent être attribués à une seule entité nosologique. Celse mentionne que l'aspect, la consistance, la taille et le comportement peuvent être variés, mais généralement ils ressemblent au carcinome. Si certaines de ces lésions sont également de vrais polypes, voire une hypertrophie des végétations adénoïdes, la plupart sont probablement des tumeurs des fosses nasales, ou des sinus, voire des néoplasies de voisinage avec extériorisation par le nez.

En ce qui concerne les tumeurs malignes, il s'agit probablement de carcinomes (carcinome du rhinopharynx ou du sinus principalement), plus rarement d'autres cancers qui surviennent dans cette localisation (lymphomes, plasmocytomes, mélanomes ou sarcomes).

Pour les tumeurs bénignes, retenons les papillomes; les hémangiomes et les lymphangiomes chez les enfants; les angiofibromes nasopharyngiens chez l'adolescent.

Dans cette liste, est-il possible de privilégier certains diagnostics?

Les tumeurs bénignes de l'enfant et de l'adolescent peuvent atteindre une taille respectable. Ils correspondent donc au tableau clinique de dilatation nasale, d'obstruction aérienne qui peut aller parfois jusqu'à mettre en jeu la vie du malade. Cette croissance progressive peut expliquer la «ressemblance avec le carcinome».

Les tumeurs malignes représentent à notre avis les diagnostics différentiels les plus probables, car elles ne se rencontrent pas seulement chez les sujets âgés. En effet, le carcinome du rhinopharynx présente deux pics de fréquence, le premier entre 15 et 25 ans et le second après 40 ans. Sa prévalence est notable dans le bassin Méditerranéen.

\section{Deuxième exemple:}

(VII, 7.14 A B) De cette affection (la cataracte), il existe plusieurs sortes. Certaines sont curables, certaines ne le sont pas. En effet, si le liquide est peu abondant, s'il ne bouge pas, s'il a la couleur de l'eau de mer ou du fer brillant et s'il laisse sur le côté quelque perception de lumière: il reste de l'espoir. Mais si le liquide est abondant, si la partie noire de l'œil, après avoir perdu sa forme naturelle en prend une autre, si la couleur du liquide est bleu sombre ou semblable à de l'or, si le liquide bouge et se déplace d'un côté ou de l'autre, on peut difficilement porter remède. Mais d'ordinaire l'affection est pire lorsqu'elle est née d'une maladie grave, de forts maux de tête ou d'un coup violent. La vieillesse n'est pas favorable aux traitements, elle qui, même sans maladie, a la vision affaiblie, et l'enfance ne l'est pas non plus, mais favorable au traitement est l'âge moyen, qui est à mi-chemin entre ces deux âges.

Il peut paraître surprenant d'utiliser un texte qui traite de la cataracte dans une discussion à propos des tumeurs. Il faut apprécier que Celse distingue 
déjà la plupart des étiologies de la cataracte. Mais parmi celles-ci, et surtout parmi les cataractes qui ont peu de chance d'être traitée c'est la cataracte de l'enfant, celle qui à une couleur semblable à l'or, où le noir de l'œil a perdu sa forme, et peut s'accompagner de maux de tête, qui concerne notre recherche. Si les cataractes congénitales sont des diagnostics différentiels possibles, il existe une tumeur de l'enfant qui présente exactement cette symptomatologie: le rétinoblastome.

Tous les signes cliniques du rétinoblastome sont en effet décrits dans ce texte, à l'exception du strabisme; mais celui-ci n'est jamais mentionné dans l'œuvre de Celse, à notre connaissance. La couleur dorée de la pupille est caractéristique du rétinoblastome et les maux de têtes sont secondaires à la croissance intracrânienne de la tumeur; la déformation de la pupille correspond à une mydriase unilatérale.

\section{Troisième texte:}

(IV, 22. 1) Parmi les maladies ${ }^{13}$ des intestins les plus proches de celles-ci sont les coliques: en grec dysenteria. L'intérieur des intestins est ulcéré: du sang s'en écoule; et ce dernier est évacué, tantôt avec de la matière fécale toujours liquide et tantôt avec des sortes de mucosités; parfois en même temps descendent des bouts de chair; le besoin d'aller à la selle est fréquent, de même qu'une douleur dans l'anus; cette douleur est accompagnée d'une évacuation en petite quantité, et cela également aggrave la douleur; cette douleur disparait après quelque temps et il y a un bref repos; mais le sommeil est interrompu, il se produit un peu de fièvre; lorsque cette maladie est devenue chronique sur une longue période, ou bien elle emporte le malade, ou bien, même si elle se termine, elle continue à le tourmenter.

Les symptômes décrits sont tous digestifs, à l'exception de l'insomnie et de petits accès de fièvre.

Le sang qui coule des intestins est attribué par Celse à une ulcération. Cette affirmation est étonnante en l'absence de radiologie et de fibroscopie. Elle devient compréhensible si l'on admet un raisonnement par analogie: les ulcères cutanés saignent, donc le saignement digestif doit provenir d'un ulcère digestif. L'aspect du sang plus ou moins mêlé avec les selles ou avec ce qui pourrait être des décharges muqueuses, des nécroses, des membranes est particulièrement bien rendu. Les autres symptômes sont: le besoin fréquent d'aller à la selle, la douleur dans l'anus accompagnée de difficulté à la défécation, descriptions qui correpondent sans doute à la fois à des ténesmes et à des faux-besoins. Le texte se termine exceptionellement par un élément pronostique très important: il ne s'agit pas d'une maladie aiguë. Elle peut conduire

13 Il s'agit de la maladie appelée simplement «maladie qui est située dans le gros intestin, particulièrement dans sa partie qui est fermée» (Livre IV,21). La description donnée par Celse correspond à un tableau de douleurs abdominales fort peu spécifiques. Le caractère paroxistique de la douleur s'accorde toutefois avec la «colique» moderne. 
à la mort ou se terminer, mais jamais complètement. Ce dernier élément exclut presque à coup sûr une origine infectieuse.

Il s'agit ici d'un tableau clinique particulièrement cohérent qui évoque principalement les maladies inflammatoires du tube digestif, et notamment la rectocolite ulcéro-hémorragique. On ne doit cependant pas exclure une tumeur colique et particulièrement anorectale. La plupart des symptômes sont en effet également évocateurs. C'est ici davantage le pronostic qui nous oriente vers une maladie de type inflammatoire.

Avec ces trois textes, il est possible d'illustrer plusieurs pespectives. D'abord, les localisations des lésions suspectes d'être des tumeurs sont très variées. Les maladies externes bien sûr, aisément accessibles à la vue et au toucher des anciens médecins, mais encore les maladies internes sont très souvent décrites avec assez de symptômes et de cohérence pour qu'il soit possible de défendre un diagnostic différentiel sérieux. Et parmi ces diagnostics, de nombreuses néoplasies représentent des hypothèses acceptables. Dans notre travail, plus de la moitié des textes retenus concernent en effet des tumeurs internes. Il faut souligner aussi que l'ensemble des âges de la vie est représenté dans le De Medicina, même si les maladies strictement pédiatriques sont rarement citées. Cette remarque a son importance si on évoque la «nouveauté» du cancer. Car il n'existe aucun argument qui permettrait d'expliquer pourquoi les enfants et les adolescents n'auraient pas aussi souffert de cancers. Les deux premiers exemples choisis s'appliquent d'ailleurs à de jeunes malades.

Alors, le cancer ou plutôt les cancers sont-ils des maladies nouvelles?

L'abondance des descriptions littéraires et la fréquence des exemples paléopathologiques de néoplasies prouve le contraire ${ }^{14}$. C'est davantage la conceptualisation de la maladie tumorale et la description de nombreuses entités cliniques qui en découle, entités certainement déjà présentes, mais inaccessibles à la compréhension du médecin de l'Antiquité, qui est responsable de cette «nouveauté», comme c'est aussi le cas pour beaucoup d'autres catégories nosologiques. Cette «nouveauté» s'applique principalement aux tumeurs internes et correspond à la première situation historique proposée par Grmek, car les tumeurs externes décrites par Celse, si elles ne peuvent être comprises de manière identique par le médecin ancien et le médecin moderne, représentent néanmoins souvent une maladie néoplasique bénigne ou maligne.

Et que faut-il penser de la prévalence du cancer? Est-elle également si différente de l'Antiquité à l'époque contemporaine qu'elle puisse être aussi à

14 Pahl WM: Tumors of bone and soft tissue in Ancient Egypt and Nubia: a synopsis of the detected cases. International Journal of Anthropology 1986;3:267-276. 
l'origine de cette «nouveauté»? Si la morbidité et la mortalité secondaires au cancer ont pris des proportions sans doute inconnues auparavant, il semble cependant inexact, à notre avis, de déclarer encore que les néoplasies étaient auparavant des pathologies exceptionnelles. Il serait alors improbable qu'un si grand nombre de tableaux cliniques compatibles avec des cancers soient réunis et décrits de manière cohérente. Pour reconnaître une maladie, il faut généralement soit qu'elle se manifeste avec une certaine fréquence, soit qu'elle présente un tableau clinique si particulier que son observation puisse difficilement être négligée, soit que le médecin dispose de moyens d'investigations plus performants que ceux de l'Antiquité. Cela est d'autant plus vrai pour le cancer qui englobe une foule de maladies diverses avec des symptômes innombrables et souvent peu spécifiques.

Plutôt que de parler de «maladie nouvelle», il serait possible de considérer le cancer - du moins la plupart d'entre eux, car il n'est pas exclu que certaines tumeurs avaient une fréquence proche voire supérieure à celle d'aujourd'hui (p.ex. les cancers ORL) - comme une «maladie émergente». Grmek a également présenté un classement de ces maladies ${ }^{15}$, qui s'applique aux maladies infectieuses. Nous proposons d'ajouter une catégorie pour les maladies tumorales: maladies dont la réalité clinique est certaine bien avant l'époque contemporaine et dont la plupart ont probablement été décrites par les médecins anciens, mais dont les modifications à la fois de la prévalence et de la conceptualisation contribuent à leur perception comme fléaux modernes.

S'il demeure difficile d'évoquer de manière globale l'émergence dans les sociétés contemporaines d'une pathologie comme le cancer, si variée dans ses localisations et ses présentations cliniques, l'appréciation, également complexe, de l'impact du cancer dans l'Antiquité nécessite une étude systématique des textes médicaux anciens qui pourront être comparés avec les témoignages de toutes origines, notamment ceux de la paléopathologie et des représentations figurées.

15 Grmek MD: Le concept de maladie émergente. Hist. Phil. Life Sci. 1993; 15: 281-296. 